\title{
Deficiency of the ER-stress-regulator MANF triggers progressive outer hair cell death and hearing loss
}

\author{
Anni Herranen', Kuu Ikäheimo', Tuuli Lankinen', Emmi Pakarinen², Bernd Fritzsch $\mathbb{1}^{3}$, Mart Saarma $\mathbb{0}^{2}$, \\ Maria Lindahl ${ }^{2}$ and Ulla Pirvola (1)
}

\begin{abstract}
The non-conventional neurotrophic factor mesencephalic astrocyte-derived neurotrophic factor (MANF) is an endoplasmic reticulum (ER)-resident protein that promotes ER homeostasis. MANF has a cytoprotective function, shown in the central nervous system neurons and pancreatic beta cells. Here, we report that MANF is expressed in the hair cells and neurons and in selected non-sensory cells of the cochlea and that Manf inactivation triggers upregulation of the ER chaperones in these cells. However, Manf inactivation resulted in the death of only outer hair cells $(\mathrm{OHCs})$, the cells responsible for sound amplification in the cochlea. All OHCs were formed in Manf-inactivated mice, but progressive $\mathrm{OHC}$ death started soon after the onset of hearing function. The robust $\mathrm{OHC}$ loss was accompanied by strongly elevated hearing thresholds. Conditional Manf inactivation demonstrated that MANF has a local function in the cochlea. Immunostainings revealed the upregulation of CHOP, the pro-apoptotic component of the unfolded protein response (UPR), in Manf-inactivated OHCs, linking the UPR to the loss of these cells. The phenotype of Manf-inactivated OHCs was distinctly dependent on the mouse strain, such that the strains characterized by early-onset age-related hearing loss (C57BL/6J and CD-1) were affected. These results suggest that Manf deficiency becomes detrimental when accompanied by gene mutations that predispose to hearing loss, by intensifying ER dyshomeostasis. Together, MANF is the first growth factor shown to antagonize ER stress-mediated $\mathrm{OHC}$ death. MANF might serve as a therapeutic candidate for protection against hearing loss induced by the ER-machinery-targeting stressors.
\end{abstract}

\section{Introduction}

Mesencephalic astrocyte-derived neurotrophic factor (MANF) belongs to the large family of neurotrophic factors (NTFs), which regulate the life and death of both neuronal and non-neuronal cells. MANF was first characterized as a factor promoting the survival of midbrain dopamine neurons in vitro $^{1}$. Later, the delivery of recombinant MANF protein to the rodent brain revealed neuroprotection against traumas ${ }^{2,3}$. Analysis of the Manf loss-of-function mutant mice has shown that the insulin-

\footnotetext{
Correspondence: Ulla Pirvola (ulla.pirvola@helsinki.fi)

${ }^{1}$ Molecular and Integrative Biosciences Research Programme, University of Helsinki, 00014 Helsinki, Finland

${ }^{2}$ Institute of Biotechnology, HILIFE Unit, University of Helsinki, 00014 Helsinki, Finland

Full list of author information is available at the end of the article.

Edited by A. Verkhatsky
}

producing beta cells of the pancreas are particularly sensitive to Manf deficiency; they die from the juvenile stages onward, leading to diabetes ${ }^{4}$. The mouse strain has an impact on the Manf knock out (KO) phenotype: KO mice under the C57BL/6J (BL6) background die perinatally due to respiratory problems, whereas most KOs under the CD-1 background survive to adulthood ${ }^{4,5}$. Thus, available data give evidence that MANF promotes cell survival in a cell context-dependent and genetic backgrounddependent fashion.

MANF resides in the endoplasmic reticulum (ER) where it promotes protein-folding homeostasis ${ }^{6}$. MANF interacts physically with the major ER chaperone, the $78 \mathrm{kDa}$ glucose-regulated protein (GRP78; also known as the binding immunoglobulin protein, $\mathrm{BiP})^{7,8}$. Proteostasis defects trigger GRP78 and MANF upregulation, in

\section{(c) The Author(s) 2020}

(c) (i) Open Access This article is licensed under a Creative Commons Attribution 4.0 International License, which permits use, sharing, adaptation, distribution and reproduction in any medium or format, as long as you give appropriate credit to the original author(s) and the source, provide a link to the Creative Commons license, and indicate if changes were made. The images or other third party material in this article are included in the article's Creative Commons license, unless indicated otherwise in a credit line to the material. If material is not included in the article's Creative Commons license and your intended use is not permitted by statutory regulation or exceeds the permitted use, you will need to obtain permission directly from the copyright holder. To view a copy of this license, visit http://creativecommons.org/licenses/by/4.0/. 
conjunction with the activation of the unfolded protein response (UPR) and calcium release from the ER stores ${ }^{9,10}$. Besides promoting ER homeostasis and cell survival in a cell-autonomous fashion ${ }^{6,11,12}$, MANF has been suggested to function non-cell-autonomously as a secreted protein via still unclear extracellular mechanisms ${ }^{7,13,14}$.

Cochlear hair cells are mechanoreceptor cells that receive, amplify, and transduce acoustic stimuli. Inner hair cells (IHCs) are responsible for the sensory transmission to the brain, via the innervating spiral ganglion (SG) neurons. Outer hair cells (OHCs) amplify acoustic signals and are thereby critical for normal hearing sensitivity. OHCs are vulnerable to various stressors and their dysfunction or death is a common reason for sensorineural hearing loss. $\mathrm{OHC}$ vulnerability differs between mouse strains. For example, the BL6 and CD-1 mice display early-onset agerelated hearing loss, as opposed to the $\mathrm{CBA} / \mathrm{Ca}$ mice $^{15-18}$. ER stress has been previously linked to hair cell loss and hearing loss ${ }^{15,19,20}$. There is no available data on the role of MANF in the inner ear. We have here focused on MANF's expression in this tissue, on its impact on cell survival and on the underlying mechanisms. This knowledge is needed for understanding the role of ER homeostasis in auditory physiology and pathophysiology.

\section{Materials and methods \\ Experimental animals \\ Manf KO mouse line}

The original Manf KO mouse line was maintained under the CD-1 background (Envigo, RRID:MGI:5658486) ${ }^{4}$. In order to avoid early-onset hearing loss that characterizes the CD-1 strain ${ }^{15,16}$, we crossed Manf heterozygous mice with the CBA/Ca strain (Envigo, RRID:MGI:2159826), which maintains good hearing until old age ${ }^{18}$. We analyzed cochleas of Manf KOs and wildtypes of the F2 generation of CD- $1 \times \mathrm{CBA} / \mathrm{Ca}$ hybrids. Manf $\mathrm{KO}$ mice suffer from diabetes ${ }^{4}$ and due to poor health, they could not be maintained beyond 8 weeks of age. The following ages were used for cochlear analysis: postnatal day 2 (P2), P12, and 4 and 8 weeks. Mice of both sexes were included in the analysis. We used heterozygous mice of this Manf KO line for X-gal histochemistry to study Manf expression, because the Manf $\mathrm{KO}$ allele includes a LacZ gene construct. It was inserted between exons 2 and 3, with an efficient splicing acceptor ensuring $\beta$-galactosidase expression controlled by the Manf promoter $^{4}$.

\section{Manf conditional mouse line}

To obtain Manf ${ }^{\text {flox/flox }}$;Pax2-Cre conditional KO (cKO) mice, we crossed the Manf flox/flox and Pax2-Cre lines ${ }^{4,21}$, both under the BL6 background (C57BL/6JRccHsd, RRID: MGI:6151402, Envigo). Pax2 is expressed in the embryonic otic placode that gives rise to various epithelial cell types and neurons of the inner ear. It is also expressed in some other tissues, such as the developing midbrain-hindbrain boundary, and the developing and adult kidney ${ }^{21}$. We studied the cochlear phenotype of the cKO mice and littermate controls $\left(\mathrm{Manf}^{+/+}\right.$;Pax2-Cre or Manf $f^{f l o x /+}$ ) before 11 weeks of age, because the BL6 mice are affected by early-onset hearing loss, the first signs of which can be seen at 3 months of age ${ }^{17}$. The following age groups, comprising both females and males, were studied: P2, P12, 5 weeks, and 8-to-11 weeks.

Blood samples were obtained from the tail vein and assayed for glucose concentration (MediSense Precision Xtra device, Abbott).

All animal work was conducted according to relevant national and international guidelines. Approval for animal experiments was obtained from the National Animal Experiment Board.

\section{Auditory brainstem response (ABR)}

We anaesthetized mice via intraperitoneal injections of ketamine hydrochloride $(75 \mathrm{mg} / \mathrm{kg}$, Ketaminol vet, Intervet International Inc.) and medetomidine hydrochloride $(1 \mathrm{mg} / \mathrm{kg}$, Domitor vet, Orion Corporation). Mice were placed on a hot water bath to maintain body temperature at $37^{\circ} \mathrm{C}$, in an electrically and acoustically shielded chamber with echo damping padding. Subdermal needle electrodes were inserted at the vertex (active), below the right ear (reference), and below the pinna of the left ear (ground). MF1 Multi-field Magnetic Speaker was used to deliver monophasic click stimuli $(0.1 \mathrm{~ms}$ duration, presented at a rate of $21 / \mathrm{s}$ ) or $4-45 \mathrm{kHz}$ tone-pip stimuli (1 ms duration, cosine-squared gated, presented at a rate of $21 / \mathrm{s}$ ). The mouse was in a free-field setup with the speaker placed $10 \mathrm{~cm}$ from the right ear. Stimuli were presented from 90 to $10 \mathrm{~dB}$ sound pressure level (dB SPL) in $5 \mathrm{~dB}$ SPL steps. Electrical responses were obtained using the RA4LI headstage (20× gain amplification) directly connected to the RA4PA Medusa preamplifier/ digitizer (250× amplification), which output to the RZ6 multi-I/O processor via fiber-optic cable. The responses were filtered (band-pass filter $300 \mathrm{~Hz}-3 \mathrm{kHz}$ ) and averaged (512 responses at each stimulus presentation level) using BioSigRZ software. The equipment was from Tucker-Davis Technologies (System 3). Thresholds were determined by visual inspection for the lowest sound intensity at which a repeatable waveform can be obtained. Calibration was performed with PCB-378C01 calibration microphone with model 480C02 sensor signal conditioner (PCB Piezotronics Inc.) using BioSigRZ and RPvdsEx virtual design studio software (version 84, Tucker-Davis Technologies).

\section{Cochlear whole mounts and immunostaining}

We perilymphatically perfused cochleas with $4 \%$ paraformaldehyde (PFA), pH 7.4, in phosphate-buffered saline (PBS). This was followed by immersion in PFA for $2 \mathrm{~h}$ at 
room temperature or overnight at $+4{ }^{\circ} \mathrm{C}$. Cochleas were then decalcified with $0.5 \mathrm{M}$ ethylenediaminetetraacetic acid (EDTA), pH 7.5, overnight at $+4{ }^{\circ} \mathrm{C}$. Organ of Corti and stria vascularis were dissected for whole mount specimens. Microdissection for the organ of Corti whole mounts has been previously described in Anttonen et al. ${ }^{22}$. Specimens were blocked with $10 \%$ goat serum (Jackson ImmunoResearch) in PBS containing 0.25\% Triton-X-100 (PBS-T) for $1 \mathrm{~h}$ and then incubated with the primary antibody cocktail in PBS-T for $48 \mathrm{~h}$ at $+4{ }^{\circ} \mathrm{C}$. We used the following primary antibodies in double-labeling: rabbit polyclonal myosin7a (Myo7a; Proteus Biosciences Cat\# 25-6790, RRID:AB_10015251, used at 1:3000), rabbit polyclonal MANF (Icosagen AS Cat\# 310-100, RRID: AB_11135308, 1:500), rabbit monoclonal GRP78 (Abcam Cat\# ab108615, RRID:AB_10890641, 1:1000), rabbit polyclonal calreticulin (Thermo Fisher Scientific Cat\# PA3-900, RRID:AB_325990, 1:200), mouse monoclonal C-terminal-binding protein 2 (CtBP2; BD Biosciences Cat\# 612044, RRID:AB_399431, 1:200), mouse monoclonal C/EBP homologous protein (CHOP; Novus Cat\# NB600-1335SS, clone 9C3, RRID:AB_11033903, 1:100), and chicken polyclonal neurofilament heavy-chain (NF-H; Millipore Cat\# AB5539, RRID:AB_11212161, 1:10,000). Primary antibodies were detected using AlexaFluor 488/ 594/647-conjugated goat anti-rabbit/mouse/chicken IgG or IgY secondary antibodies (Invitrogen). After antibody incubations, we visualized F-actin filaments using Oregon Green 514-conjugated or Rhodamine 568-conjugated phalloidin (Invitrogen). Nuclei were stained with DAPI. ProLong Gold anti-fade reagent was used for mounting (Invitrogen).

\section{Paraffin sections and histochemistry}

We fixed and decalcified cochleas as above. We then embedded the specimens into paraffin (Histoplast IM, Thermo Fisher Scientific). We cut them in a midmodiolar plane into 5 - $\mu \mathrm{m}$-thick sections. Epitope retrieval was done by microwave heating $(900 \mathrm{~W})$ in $10 \mathrm{mM}$ citrate buffer, $\mathrm{pH}$ 6.0, with a boiling time of $11 \mathrm{~min}$. Sections were blocked with $10 \%$ goat or horse serum (Jackson ImmunoResearch) in PBS-T for $1 \mathrm{~h}$ and then incubated with primary antibodies for $48 \mathrm{~h}$ at $+4{ }^{\circ} \mathrm{C}$. We used the following primary antibodies: rabbit monoclonal GRP78 (Abcam, used at 1:10,000); rabbit monoclonal protein disulfide isomerase (PDI; Cell Signaling Technology Cat\# 3501, RRID:AB_2156433, 1:1000), and goat polyclonal prestin (Santa Cruz Biotechnology Cat\# sc-22694, RRID: AB_2190502, 1:1000). Detection was done with the Vectastain Elite $\mathrm{ABC}$ kit and diaminobenzidine substrate (DAB Detection kit). Sections were counterstained with methyl green. Some sections were used for hematoxylin-eosin staining (Hematoxylin and Eosin Stain Kit) (all from Vector Laboratories). Sections were mounted with Permount (Thermo Fisher Scientific). The intensity of immunostaining was compared between control and mutant sections that had been processed and imaged exactly in the same way.

\section{Preadsorption assay}

To confirm the lack of non-specific GRP78 staining, we incubated the primary antibody (1:6000 dilution; $0.16 \mu \mathrm{g} / \mathrm{ml})$ with a 2.5 -fold excess $(0.4 \mu \mathrm{g} / \mathrm{ml})$ of the immunizing peptide (Abcam) overnight at $+4{ }^{\circ} \mathrm{C}$. We then applied this mix on cochlear sections. The preadsorption abolished all staining in sections (See Results Fig. 4e).

\section{Imaging}

BX61 microscope equipped with UPlanApo $\times 10$ (NA 0.4 ) and $\times 40$ (NA 1.3, oil immersion) objectives was used for transmitted light images. They were taken with the DP73 CCD color camera and CellSens software (all from Olympus). Further image processing was performed in Adobe Photoshop CC 2017.

\section{X-gal staining}

We dissected cochleas from heterozygote mice of the Manf $\mathrm{KO}$ line and perilymphatically perfused cochleas with $4 \%$ PFA, followed by immersion in the fixative at room temperature for $1.5 \mathrm{~h}$. X-gal incubation was performed at $+37^{\circ} \mathrm{C}$ for $15 \mathrm{~h}$. Cochleas were thereafter postfixed, decalcified, and processed for whole mounts or paraffin sections as earlier described in Herranen et al. ${ }^{23}$. In paraffin-embedding, deparaffination and dehydration of sections, the time in each alcohol step was kept to a minimum to avoid fading of X-gal staining. Sections were counterstained with $0.1 \%$ Nuclear Fast Red and mounted in Permount. Specimens were imaged with the BX61 microscope setup described above.

\section{Cochlear mapping and hair cell and synaptic ribbon counts Total and regional $\mathrm{OHC}$ loss counts}

We prepared a frequency map from each cochlea using the Measure Line.class ImageJ plugin (available from Eaton-Peabody Laboratories Histology Resources) and the calculations were done according to Müller et al. ${ }^{24}$. OHC loss was counted from DAPI/phalloidin/Myo7a/CtBP2stained whole mounts. Lack of both the nucleus and the positively stained cell body was used as the criterion for the absence of a cell. OHC loss was counted across the cochlear duct, from the basalmost hook region to the apex. Image stack acquisition for cell counts was performed with Axio Imager.M2 microscope equipped with Apotome 2 structured illumination slider, using PlanApo $\times 10$ objective (NA 0.45 ) and a $z$-step-size of $1.5 \mu \mathrm{m}$, CMOS camera (Hamamatsu ORCA Flash 4.0 V2) and Zen 2 software (all from Zeiss). Percentage of lost OHCs was counted from the total $\mathrm{OHC}$ population. In addition, 
8-to-10-week old control and cKO mice were used to count the percentage of $\mathrm{OHC}$ loss at the 16, 32, and $45 \mathrm{kHz}$ frequency regions. The frequency-specific cell counts were done from $\times 40$ image stacks where the countable area included $\sim 140$ OHCs.

\section{Presynaptic ribbon counts}

Synaptic ribbons in the basal pole of IHCs were counted from the same whole mounts as used for $\mathrm{OHC}$ loss quantification, using the CtBP2 antibody to mark the synaptic ribbons. Ribbon quantification per IHC was performed at 16, 32, and $45 \mathrm{kHz}$ cochlear regions from the maximum intensity projection images, which were processed with ZEN 2 software. $z$-stacks were obtained with Axio Imager.M2 microscope equipped with Apotome 2 structured illumination slider, using PlanApo $\times 40$ objective (NA 1.3, oil immersion) and a $z$-step-size of $0.25 \mu \mathrm{m}$. The Hamamatsu ORCA Flash $4.0 \mathrm{~V} 2$ camera was used for image acquisition. Each image stack spanned from the cuticular plate to the synaptic pole of IHCs and contained the entire synaptic pole of $\sim 40$ IHCs.

\section{Statistical analysis}

For all statistical analyses Origin 2018b (OriginLab Corporation) was used. Significant differences were reported with 95\% confidence intervals. Error bars represent standard deviation (SD) except in ABR measurements where standard error of mean (SEM) was used. Welch's $t$-test was used to compare the means of two experimental groups. One-way or two-way ANOVA with Tukey's test was utilized for means comparison of multiple groups.

For the Manf $\mathrm{KO}$ line, $\mathrm{KO}$ and wildtype males were used for the comparison of the body weight and blood glucose concentration. In case of the cKO line, females were used for these assays. The body weight and glucose concentration were compared with Welch's $t$-test (cKO) and one-way ANOVA (KO).

Two-way ANOVA was utilized for comparing the mean of ABR thresholds, OHC loss and synaptic ribbon numbers per IHC. ABR thresholds were compared between control and Manf KO or cKO mice at 4/5 weeks and at 8/ 8-11 weeks of age, respectively, in response to click and tone-pip stimuli. In the Manf KO line under CD-1 $\times \mathrm{CBA} /$ Ca background, ABR thresholds and OHC loss of the $s$ and $l$-groups of KOs (see the section "Results") were compared to wildtypes. Synaptic ribbon counts per IHC were compared between control and Manf cKO mice at three cochlear frequency regions.

\section{Results}

MANF is expressed in distinct cell types of the cochlea

We used X-gal histochemistry and immunostaining to study MANF expression in the cochlea (Fig. 1a-o). We benefited from the design of the Manf $\mathrm{KO}$ allele $\mathrm{e}^{4}$, including a $L a c Z$ gene construct that we detected by X-gal staining in heterozygote mice from the Manf $\mathrm{KO}$ line. $\mathrm{X}$-gal staining was seen in the adult SG neurons and hair cells, IHCs showing stronger staining than OHCs. Epithelial cells lateral to the organ of Corti and interdental cells on the modiolar side were also stained (Fig. 1a, a', d). This staining pattern was visible throughout the length of the spiraling cochlea. We confirmed the absence of nonspecific X-gal staining in wildtype cochleas (Fig. 1a").

To study Manf expression at younger ages, heterozygote cochleas were analyzed at P2, P12, and at 4 weeks of age. $\mathrm{X}$-gal staining was relatively weak in the neonatal cochlea, but became stronger by $\mathrm{P} 12$, the stage representing the onset of hearing function. The staining intensity was maintained at this level at later ages (Fig. 1i-n). Marginal cells of the stria vascularis showed very weak staining at all ages studied (Fig. 10; data not shown).

We performed MANF immunohistochemistry on cochlear whole mounts from wildtype mice. MANF was strongly expressed in the adult SG neurons and IHCs, and weaker in OHCs (Fig. 1b, f-h), in accordance with X-gal staining. MANF staining was detected throughout the IHC cytoplasm, whereas it was concentrated to the region just below the cuticular plate in OHCs (Fig. 1f, $g^{\prime}$ ). We did not detect immunostaining in Manf KO cochleas, confirming the specificity of the antibody used (Fig. 1c, h). These findings are in line with prior data that MANF is an ER-resident protein ${ }^{6}$ and that ER is accumulated to the pericuticular region in $\mathrm{OHCs}^{25}$.

\section{Manf KO mice show progressive outer hair cell loss and elevated hearing thresholds}

The original Manf $\mathrm{KO}$ mouse line was maintained under the CD-1 background ${ }^{4}$. CD-1 mice suffer from early-onset hair cell loss and hearing loss ${ }^{15,16}$. To reduce this genetic background effect, we crossed the Manf $\mathrm{KO}$ line with the CBA/Ca strain and analyzed F2 hybrids. We found that wildtype hybrids have a complete $\mathrm{OHC}$ population and their ABR thresholds are within the normal range, making the comparison to $\mathrm{KO}$ littermates feasible.

We analyzed cochleas of Manf KO mice and wildtype littermates under the hybrid background at P12 and at 4 and 8 weeks of age. Cytocochleograms from KOs at P12 showed the presence of all hair cells (Fig. 2a, $\mathrm{a}^{\prime}$ ). However, a part of KOs displayed prominent $\mathrm{OHC}$ loss by 4 weeks and this loss progressed further, evidenced by a doubling by 8 weeks. OHC loss was concentrated to the basal part of the cochlea at 4 weeks and had extended to the middle part by 8 weeks (Fig. 2b, c). In line with the robust $\mathrm{OHC}$ loss, ABR thresholds were prominently elevated (Fig. 2d). The survival of IHCs and SG neurons was unaffected (Fig. 2b", b"', e). Surprisingly, in addition to the 


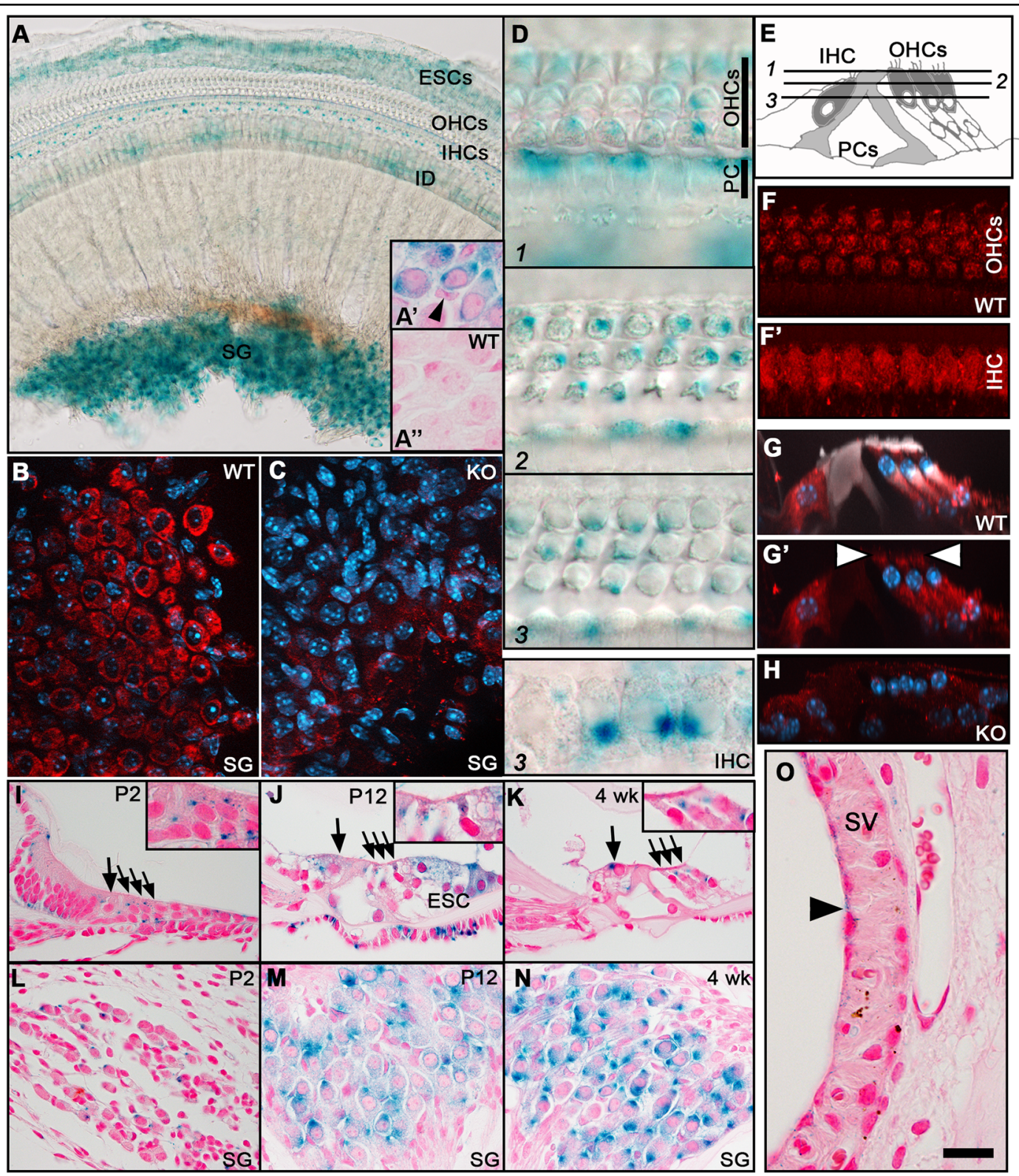

Fig. 1 MANF expression in the cochlea, revealed by X-gal histochemistry and immunostaining. a The whole mount shows prominent X-gal staining in the SG, IDs, IHCS, and ESCs. The weaker staining in OHCs is not evident in this low magnification. $\mathbf{a}^{\prime} \mathbf{X}$-gal staining is present in SG neurons and absent in the satellite cells (arrowhead), revealed in a paraffin section. $\mathbf{a}^{\prime \prime}$ X-gal staining is absent from SG neurons from a WT mouse. b MANF immunostaining (red) is prominent in SG neurons, revealed in a whole mount. DAPI marks nuclei (blue). c SG neurons from a KO mouse lack MANF staining. $\mathbf{d}$ Whole mount is viewed at different planes from the hair cell cuticular plate down to nuclear level. X-gal staining is prominent in IHCs and pillar cells (supporting cells). In OHCs, X-gal staining is concentrated to the plane below the cuticular plate (plane 2). Note, however, that X-gal staining is not a readout of intracellular protein distribution pattern. e Schematic cross-section through the organ of Corti depicts the imaging planes in $\mathbf{d}$ and the cell types. $\mathbf{f}$ Whole mount shows MANF immunostaining in OHCs, at the plane below the cuticular plate. $\mathbf{f}^{\prime}$ The same wholemount shows widespread MANF immunostaining in IHCs. g, $\mathbf{g}^{\prime}$ Maximum intensity projection of MANF-immunostained (red) whole mount viewed in transverse plane. Phalloidin (white) labels F-actin and DAPI (blue) nuclei. MANF is expressed below the cuticular plate (arrowheads) in OHCs. MANF is widely expressed in the IHC cytoplasm as well as in Deiters' cells (supporting cells) beneath OHCs. Phalloidin labels the hair cell apices and pillar cells. $\mathbf{h}$ MANF immunostaining is absent from the organ of Corti of KO mice. $\mathbf{i}, \mathbf{j}, \mathbf{k}$ Transverse paraffin sections show X-gal staining in the organ of Corti at different ages. Large arrows mark IHCs, smaller arrows the three OHC rows. Insets show OHCs in higher magnification. I, $\mathbf{m}, \mathbf{n}$ Transverse paraffin sections show the increase of X-gal-staining intensity along aging. $\mathbf{o}$ Manf is very weakly expressed in the luminally located marginal cells (arrowhead) of the stria vascularis. Abbreviations: KO knock out; WT wildtype; SG spiral ganglion; OHCs outer hair cells; IHCs inner hair cells; IDs interdental cells; ESCs external sulcus cells, PCs pillar cells, DCs Deiters' cells; SV stria vascularis. Scale bar (in o): a $60 \mu \mathrm{m} ; \mathbf{b}, \mathbf{c} 20 \mu \mathrm{m} ; \mathbf{d} 10 \mu \mathrm{m} ; \mathbf{f}^{\prime} \mathbf{f}^{\prime}, \mathbf{g}, \mathbf{g}^{\prime}$, h, o $15 \mu \mathrm{m} ; \mathbf{i}-\mathbf{n} 30 \mu \mathrm{m}$. 

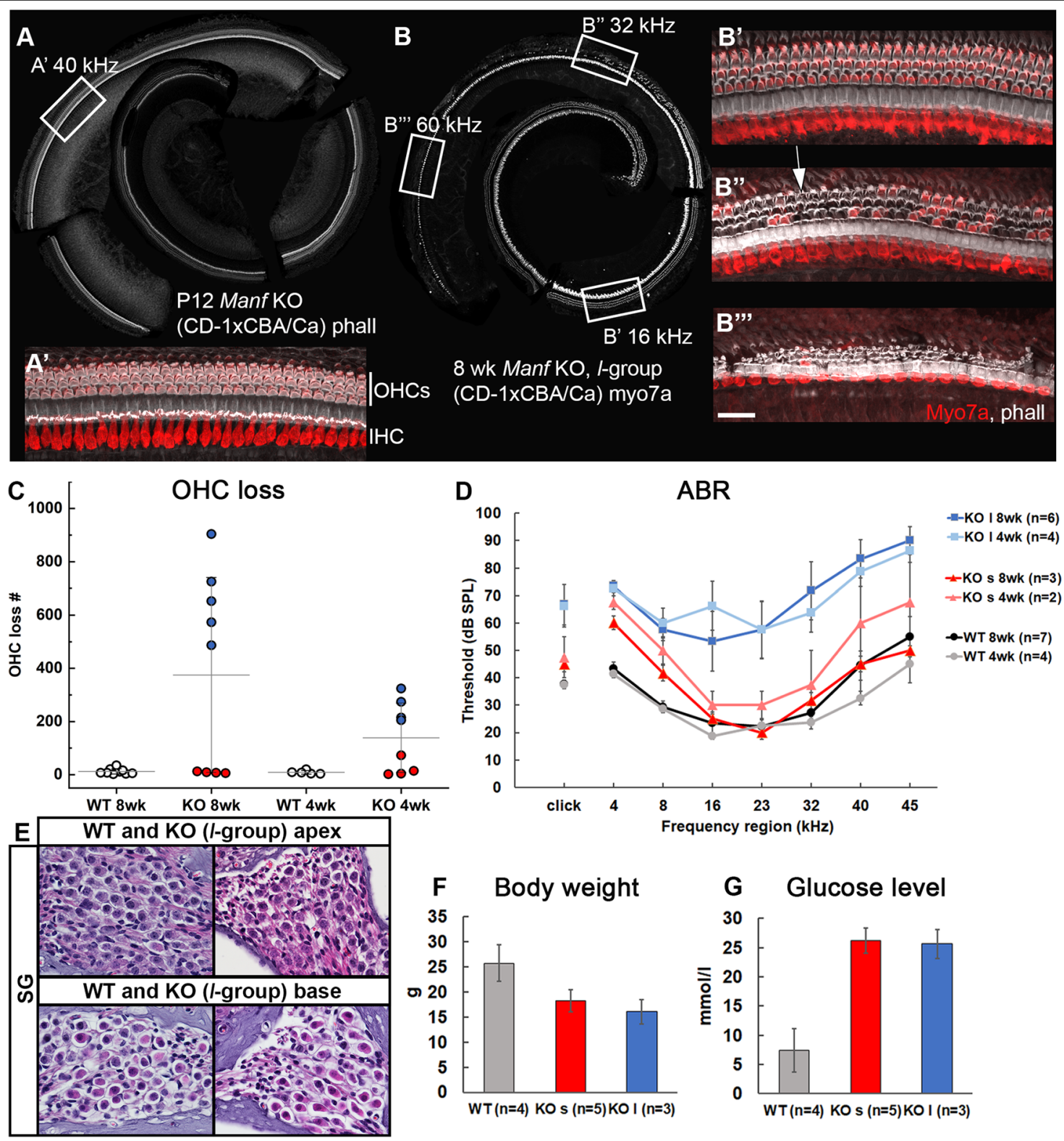

Fig. 2 Manf KO mice under the CD-1 $\times$ CBA/Ca hybrid background show progressive OHC loss in a background-dependent fashion. $a_{1} a^{\prime}$ All hair cells are present in the $\mathrm{KO}$ cochlear whole mount at P12, revealed by phalloidin labeling. Boxed area in a marks the $40 \mathrm{kHz}$ region, shown in a higher magnification in $\mathbf{a}^{\prime}$, with phalloidin (white) and myo7a (red) labeling. Phalloidin marks the apex of hair cells as well as pillar cells located between IHCs and OHCs. Myo7a marks the hair cell cytoplasm. b At 8 weeks of age, l-group of KO mice shows robust OHC loss from the 60 to $20 \mathrm{kHz}$ region in the tonotopic axis, revealed by myo7a-staining (white). Boxed areas in $\mathbf{b}$ mark the frequency regions shown in higher magnifications in $\mathbf{b}^{\prime}-$ $\mathbf{b}^{\prime \prime \prime}$, with phalloidin (white) and myo7a (red) labeling. $\mathbf{b}^{\prime}$ All OHCs are present in the $16 \mathrm{kHz}$ region. $\mathbf{b}^{\prime \prime}$ A large part of OHCs are lost in the $32 \mathrm{kHz}$ region. They are replaced by phalloidin-labeled F-actin scars (arrow in $\mathbf{b}^{\prime \prime \prime}$ ), formed by Deiters' cells. $\mathbf{b}^{\prime \prime \prime}$ Most OHCs are lost in the $60 \mathrm{kHz}$ region. Note that IHCS are present in this otherwise damaged organ of Corti. c Scatter plot with mean \pm SD of OHC loss in KO and WT cochleas. KO cochleas are split into the $s$ - and l-groups (red and blue dots, respectively), based on the extent of OHC loss. Each dot represents one mouse. $\mathbf{d}$ At 4 and 8 weeks of age, the difference in ABR thresholds between the KO I-group mice and WT littermates is statistically significant at all frequencies tested (two-way ANOVA, Tukey's test, $P<0.05)$. This is also the case in the response to click stimuli $(P<0.05)$. ABR thresholds are comparable between the KO s-group and WTs at both age groups and at all frequencies tested, except at $4 \mathrm{kHz}$ (two-way ANOVA, Tukey's test, $P<0.01$ ). Error bars represent SEM. e Hematoxylin-eosin-stained paraffin sections show a normal complement of SG neurons in the apex and base of the cochlea from the KO l-group. $\mathbf{f}, \mathbf{g}$ The decrease in body weight and increase in blood glucose concentration are statistically significant between KOs and WT littermates. Males were used for analysis. Note that this difference applies to both the 1 - and s-group of KOs (one-way ANOVA, Tukey's test, $P<0.001$ for all comparisons). Abbreviations: KO knock out, WT wildtype; phall phalloidin; myo7a myosin7a; IHC inner hair cell, OHCs outer hair cells; ABR auditory brainstem response; SG spiral ganglion. Scale bar (in $\mathbf{b}^{\prime \prime \prime}$ ): $\mathbf{a}, \mathbf{b} 200 \mu \mathrm{m} ; \mathbf{a}^{\prime}, \mathbf{b}^{\prime}-\mathbf{b}^{\prime \prime \prime} 25 \mu \mathrm{m}$; e $35 \mu \mathrm{m}$. 
KOs with strongly affected $\mathrm{OHC}$ survival and ABRs, we found $\mathrm{KO}$ individuals with practically no change in these auditory parameters and, thus, they were comparable to wildtypes (Fig. 2c, d). Thereby, Manf KO mice formed two non-overlapping groups, the large and small group $(l-$ and $s$-group based on the extent of $\mathrm{OHC}$ loss and ABR rise (Fig. 2c, d). At 8 weeks, on average $668 \pm 159$ OHCs $(n=5$ mice) were lost in the $l$-group, while only $9 \pm 3$ OHCs $(n=4$ mice) were absent in the $s$-group (Fig. 2c). Compared to wildtypes, the difference in $\mathrm{OHC}$ numbers was statistically significant in the $l$-group, both at 4 and 8 weeks of age (two-way ANOVA, Tukey's test, $P<0.05$ at 4 weeks and $P<0.001$ at 8 weeks). Correspondingly, ABR thresholds were significantly elevated only in the $l$-group (Fig. 2d). Of note, both $l$ - and $s$-group mutants showed other phenotypic changes typical to the Manf KO mice, namely hyperglycemia and reduced growth (Fig. 2f, g) ${ }^{4,5}$.

These results point to genetic heterogeneity of the Manf $\mathrm{KO}$ mice under the hybrid $(\mathrm{CD}-1 \times \mathrm{CBA} / \mathrm{Ca})$ background. Support for this conclusion came from our analysis of Manf $\mathrm{KO}$ mice under the pure CD-1 strain, a strain characterized by early-onset hearing loss ${ }^{15,16}$. These KOs showed extensive $\mathrm{OHC}$ loss and, notably, all individuals showed this robust effect $(578 \pm 236, n=8$ mice, 8 weeks of age). Thus, under the hybrid background, Manf inactivation combined with the hearing loss predisposing CD1 background seem to characterize the $l$-group, while Manf inactivation together with the $\mathrm{CBA} / \mathrm{Ca}$ status characterize the $s$-group.

\section{Conditional inactivation suggests a local function for MANF in the cochlea}

To rule out the effects of hyperglycemia on $\mathrm{OHC}$ viability and to obtain more understanding of the genetic background effect, we generated Manf $f^{\text {lox/flox }}$;Pax2-Cre cKO mice where Manf is inactivated in the epithelial cells and neurons of the inner ear ${ }^{21}$. This conditional inactivation approach does not target the beta cells of the pancreas and, correspondingly, we found comparable glucose levels in $\mathrm{cKO}$ and control littermates. Also the body weight was comparable between the genotypes (Fig. 3l, m).

We maintained Manf cKO mice under the pure BL6 background. As BL6 mice show initial signs of age-related hearing loss at 3 months of age ${ }^{17}$, we restricted our analysis to younger ages. Heterozygote $\mathrm{Manf}^{\text {flox/+}}$;Pax2-Cre cochleas did not show OHC loss (data not shown). cKO mutants showed a complete hair cell population at P12. Prominent OHC loss had developed in the basal part of the cochlea by 5 weeks of age and it progressed to the middle part, based on the analysis between 8 and 11 weeks of age (Fig. 3a-c). The cell loss accounts for $\sim 25 \%$ of the total $\mathrm{OHC}$ population (about $2500 \mathrm{OHCs}$ in the mouse cochlea). Consistent with this $\mathrm{OHC}$ loss, ABR thresholds were strongly elevated (Fig. 3d). Changes in both of these parameters were similar as in the $l$-group of Manf full-KO mice. Also similar to these full-KOs, the survival of IHCs and SG neurons was unaffected, except for a few IHCs that were absent in the region of strongest $\mathrm{OHC}$ loss (Fig. $3 \mathrm{a}-\mathrm{a}^{\prime \prime}$, data not shown). Together, MANF has a local function in the cochlea and OHCs are the major targets of Manf inactivation. Notably, as opposed to Manf KOs under the hybrid background, cKOs under the BL6 background showed a uniform cochlear phenotype.

Despite MANF expression in IHCs and SG neurons, Manf inactivation did not abrogate the survival of these cells. IHCs are connected to SG neurons by ribbon synapses, $\sim 16$ ribbons per IHC. We next asked whether the maintenance of these ribbon synapses is affected by Manf inactivation, taking into account that ER stress is linked with changes in calcium dynamics and that the ribbon synapse physiology depends on intracellular calcium buffering ${ }^{26}$. We prepared whole mounts of cKO and control cochleas between 8 and 11 weeks of age and quantified the CtBP2-positive presynaptic ribbons per IHC. cKOs showed decreased ribbon numbers in the basal portion of the cochlea, while the counts in the upper portion were comparable to controls (Fig. 3e). Thus, Manf inactivation triggered the loss of synapses between IHCs and SG neurons, and this synaptopathy was concentrated to the high-frequency region of the cochlea where also OHC loss was prominent. OHCs are also connected to SG neurons by ribbon synapses, with 2-4 ribbons per $\mathrm{OHC}$. These CtBP2-positive ribbons were maintained in surviving $\mathrm{OHCs}$ of Manf-inactivated cochleas (data not shown).

We next focused on structural aspects of Manf-inactivated $\mathrm{OHCs}$. The motor protein prestin underlies the active sound amplification by OHCs. Prestin is expressed in the $\mathrm{OHC}$ lateral wall. Immunostainings revealed that prestin is similarly expressed in OHCs of cKO and control cochleas (Fig. 3f, g). We used phalloidin labeling to reveal possible structural alterations in the $\mathrm{OHC}$ stereociliary bundles. At P12, no obvious differences were found between the genotypes (data not shown). However, adult cKOs displayed abnormal OHC bundles in the basal portion of the cochlea, showing up as absence or fusion of stereocilia (Fig. 3h, i). As OHC loss was robust in the same region, the bundle dysmorphology seems to be linked with the death-prone phenotype of Manf-inactivated OHCs.

We did not find evidence of perturbed SG neuron survival in Manf-inactivated cochleas (Fig. 1e). To rule out possible defects in the SG afferent innervation to OHCs, a defect that could promote $\mathrm{OHC}$ loss ${ }^{27}$, whole mounts of adult cochleas were stained with the NF-H antibody $^{28}$. In the middle part of cKO cochleas where a many $\mathrm{OHCs}$ were still present, the afferent innervation pattern to $\mathrm{OHCs}$ was comparable to control specimens 


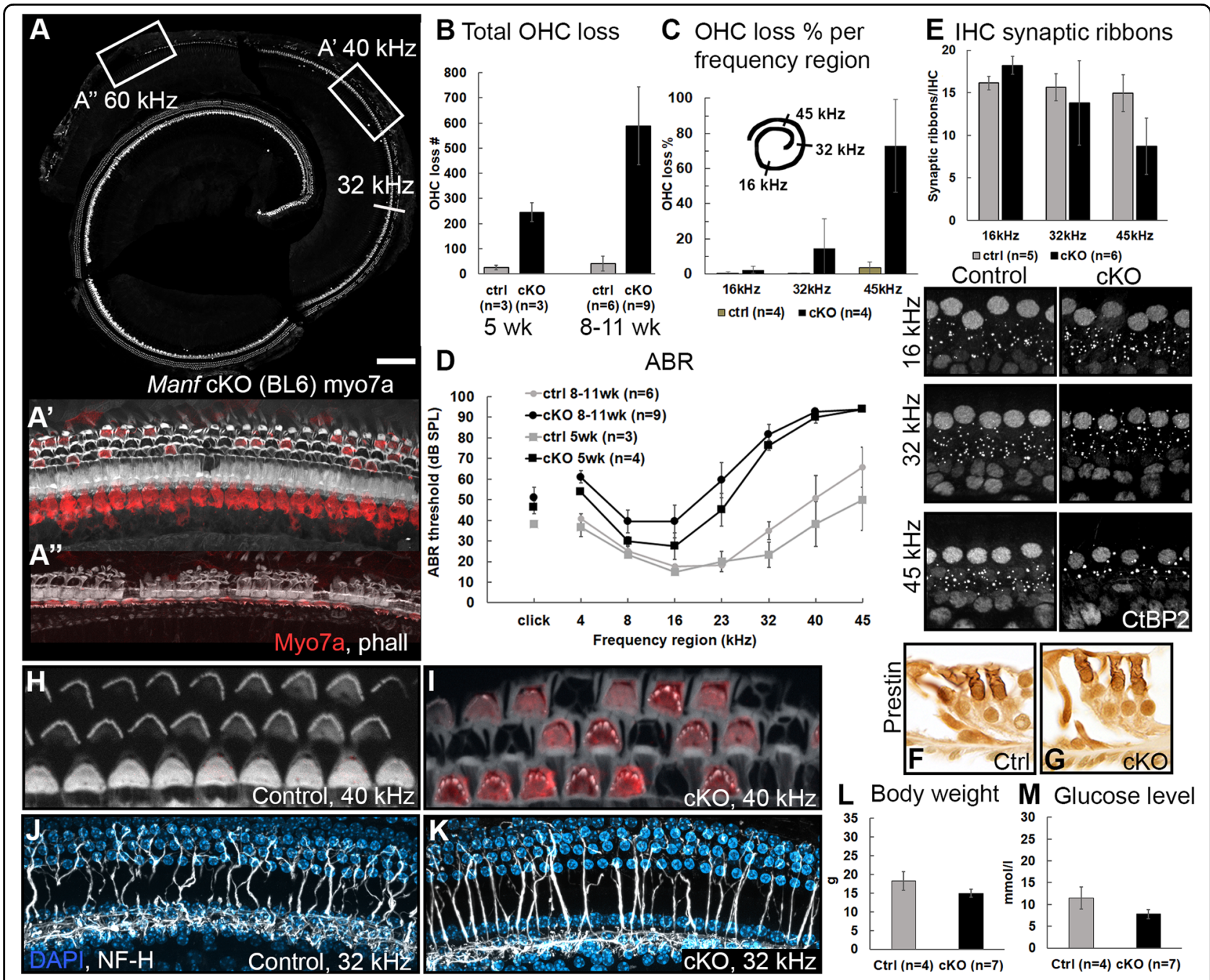

Fig. 3 Manf cKO mice under the BL6 background show progressive OHC loss. a The whole mount from a 8-week-old Manfloxflox;Pax2-Cre mouse shows extensive loss of myo7a-positive OHCs in the basal part of the cochlea. The loss continues up to the $20 \mathrm{kHz}$ region. Boxed areas represent higher magnifications shown in $\mathbf{a}^{\prime}$ and $\mathbf{a}^{\prime \prime}$. $\mathbf{a}^{\prime}, \mathbf{a}^{\prime \prime}$ A subset of OHCs is preserved at $40 \mathrm{kHz} \mathbf{a}^{\prime}$, but OHC loss is near-total at $60 \mathrm{kHz} \mathbf{a}^{\prime \prime}$, revealed by phalloidin (white) and myo7a (red) labeling. Note that also one $\mathrm{IHC} \mathbf{a}^{\prime \prime}$ and several pillar cells $\mathbf{a}^{\prime}, \mathbf{a}^{\prime \prime}$ are lost in this strongly degenerated organ of Corti. b Mean OHC loss in CKO mice at 8-to-11 weeks of age is statistically significant compared to age-matched controls (two-way ANOVA, Tukey's test, $P<0.001)$. Total OHC loss doubles in 5 weeks when comparing cKO mice at 5 and 8-to-11 weeks of age. Error bars represent SD. c The progressive $\mathrm{OHC}$ loss shown as the percentage of lost $\mathrm{OHC}$ at frequency regions 16, 32, and $45 \mathrm{kHz}$ in 8-to-10-weeks old cKO and ctrl mice. Error bars represent SD. $\mathbf{d}$ In both age groups (5 weeks and 8-to-11 weeks), the rise in ABR thresholds in KO mice is statistically significant at high-frequencies, where also OHC loss is robust, compared to controls (two-way ANOVA, Tukey's test, $P<0.05$ ). Error bars represent SEM. e Synaptic ribbon counts (mean \pm SD) in CKO mice (8-to-11 weeks of age) are comparable with age-matched controls at 16 and $32 \mathrm{kHz}$, but a statistically significant decrease in ribbon numbers is seen at $45 \mathrm{kHz}$ (two-way ANOVA, Tukey's test, $P<0.05$ ). In the three frequency regions, CtBP2-stained synaptic ribbons were counted per $\mathrm{IHC}$ from maximum intensity projections of whole mount z-stacks. $\mathbf{f}, \mathbf{g}$ Transverse paraffin sections show prestin expression in the OHC lateral wall of both genotypes. $\mathbf{h}$, $\mathbf{i}$ Whole mounts from the basal part of cochleas of cKO and control mice were labeled with phalloidin (white) and myo7a antibody (red) and imaged at the level of the OHC stereociliary bundles. The V-shaped bundles of cKOs show a degenerative appearance compared to the cohesive bundles of controls. Due to the slightly different orientation of the specimens, cytoplasmic myo7a staining is not readily seen in the control specimen. $\mathbf{j}, \mathbf{k}$ Maximum intensity projections of NF-H-stained whole mounts display afferent innervation (white) to OHCs that is comparable in CKO and control cochleas. DAPI (blue) marks nuclei. The images are from the middle part of the cochlea where OHC loss is scattered. I, m Body weight and blood glucose concentration are comparable between 8-to-11-week-old cKOs and control littermates. Females were used for this analysis (Welch's $t$-test, $P<0.05$ ). Abbreviations: $C K O$ conditional knock out; myo7a myosin7a; phall phalloidin; ABR auditory brainstem response; OHC outer hair cell; ctrl control; CtBP2 C-terminal binding protein 2; NF-H neurofilament heavy-chain. Scale bar (in a): a $175 \mu \mathrm{m} ; \mathbf{a}^{\prime}, \mathbf{a}^{\prime \prime}, \mathbf{j}, \mathbf{k} 20 \mu \mathrm{m}$; e $10 \mu \mathrm{m} ; \mathbf{f}, \mathbf{g} 25 \mu \mathrm{m} ; \mathbf{h}, \mathbf{i} 7 \mu \mathrm{m}$. 
(Fig. 3j, k). Hence, we did not find a link between afferent innervation and abrogated $\mathrm{OHC}$ viability in Manf-inactivated cochleas.

\section{Manf inactivation causes upregulation of ER stress and UPR markers in the cochlear sensory cells}

MANF physically interacts with the ER chaperone GRP78 and this complex antagonizes ER stress caused by protein folding defects ${ }^{7,8}$. To find out if GRP78 is upregulated in Manf-inactivated cochleas, similar as shown in the pancreas ${ }^{4}$, we stained cochlear sections from 8-weekold mice with the GRP78 antibody. Wildtype cochleas showed widespread, moderate expression. cKO cochleas displayed GRP78 upregulation in the cells normally expressing MANF, including OHCs, IHCs, and SG neurons (Fig. $4 \mathrm{a}-\mathrm{C}$ ). PDI, another ER stress-induced chaperone, was upregulated in the same cells (Fig. $4 \mathrm{~d}-\mathrm{g}$ ). In $\mathrm{OHCs}$ of cKO mice, these ER stress markers were concentrated to the pericuticular region, similar as the ER organelle marker calreticulin (Fig. 4h, h'). ER stress is associated with UPR activation. The pro-apoptotic UPR branch engages $\mathrm{CHOP}$ as a distal component. To find out if $\mathrm{CHOP}$ is induced in Manf-deficient OHCs, cochlear whole mounts from 8-week-old mice were stained with the CHOP antibody. As opposed to wildtype OHCs, $\mathrm{CHOP}$ was induced in a subset of OHCs in the basal part of cKO cochleas. This induction formed a front along the base-to-apex axis of the cochlea and it preceded the front of OHC loss (Fig. 4i-k). CHOP was not expressed in other cochlear cell types, consistent with the fact that cell death was confined to OHCs (Fig. 4l, data not shown).

\section{Manf deficiency does not abrogate the survival of marginal cells of the stria vascularis}

Mechanotransduction by hair cells and the viability of these cells depend on the ionic composition of the endolymph. Marginal cells of the stria vascularis secrete ions into the endolymph. Strial whole mounts and sections from Manf cKO cochleas showed distinct GRP78 upregulation in marginal cells (Fig. $4 \mathrm{~m}-\mathrm{p}$ ), consistent with Manf expression, albeit weak, in these cells in wildtype animals (Figs. $1 \mathrm{o}$ and 4s). However, phalloidinlabeled whole mounts showed a normal hexagonal pattern of marginal cell boundaries (Fig. 4q, r), indicating that Manf depletion does not disrupt the survival of these cells.

\section{Manf deficiency does not abrogate the survival of vestibular sensory cells}

Finally, we studied whether the inner ear vestibular organs are affected by Manf inactivation. Hair cells of the utricle, saccule, and cristae as well as the vestibular ganglion neurons showed MANF expression, evidenced by X-gal staining and immunostaining (Supplementary Fig. 1a-e', data not shown). However, vestibular organs of
Manf cKO and full-KO mice did not show loss of Myo7apositive hair cells and, consistently, these mutants lacked behavioral abnormalities associated with vestibular disorders, such as circling, head bobbing, and abnormal gait (Supplementary Fig. 1f, g, data not shown).

\section{Discussion}

Here we have demonstrated by a genetic approach that MANF, a growth factor promoting ER homeostasis, is required for $\mathrm{OHC}$ survival. MANF was expressed in IHCs, OHCs, SG neurons, and in selected non-sensory cells of the cochlea. Manf inactivation triggered upregulation of the ER stress markers, the chaperones GRP78 and PDI, in these cells. However, it was primarily the $\mathrm{OHC}$ survival that was affected by Manf inactivation. Analogously, the pancreatic beta cells show a tight association between Manf inactivation, ER stress, and a death-prone phenotype $^{4}$. We studied the inner ear phenotype of Manf KO mice. Because these mice suffer from diabetes, that might impair hearing function, we also generated non-diabetic Manf cKO mice. The mutants showed robust OHC loss and ABR threshold shifts. Thereby, we confirmed that MANF has a local role in the cochlea in promoting OHC survival and normal hearing function.

Local injection of the ER stress-activating drug tunicamycin into the rat cochlea has been shown to cause $\mathrm{OHC}$ death $^{19}$. More recently, transmembrane and tetratricopeptide repeat 4 (Tmtc4), a broadly expressed regulator of ER calcium dynamics, was linked to ER stress and UPR activation in the cochlea ${ }^{20}$. It was shown that ER stress and UPR markers are induced in the cochleas of Tmtc4 $\mathrm{KO}$ mice and that these mice suffer from hearing loss. These data support our results with the Manf-inactivated mice, showing that ER stress triggers OHC death and hearing loss. Cellular ER stress engages UPR activation that has an adaptive role aimed to restore proteostasis. Prolonged ER stress can activate the pro-apoptotic UPR branch with CHOP as a distal component ${ }^{9}$. We found CHOP induction in OHCs of Manf-inactivated cochleas, specifically in the area of ongoing $\mathrm{OHC}$ death. Thus, our results link the upregulation of ER chaperones and UPR signaling to $\mathrm{OHC}$ death. In wildtype OHCs, MANF had a distinct subcellular distribution, being expressed in the neck region beneath the cuticular plate. Prior studies have localized accumulation of ER and mitochondria to this region ${ }^{25,29}$. Correspondingly, we found GRP78 and PDI upregulation specifically in the neck region of Manf-deficient OHCs. Recently, we utilized serial block-face scanning electron microscopy to study trauma-induced pathology in the cochlear sensory epithelium $^{22}$. In addition to that we localized mitochondria and cisternae to the OHC's neck region, we found that this region becomes vacuolized in the late phases of degeneration. The $\mathrm{OHC}$ is then decapitated at this site, 

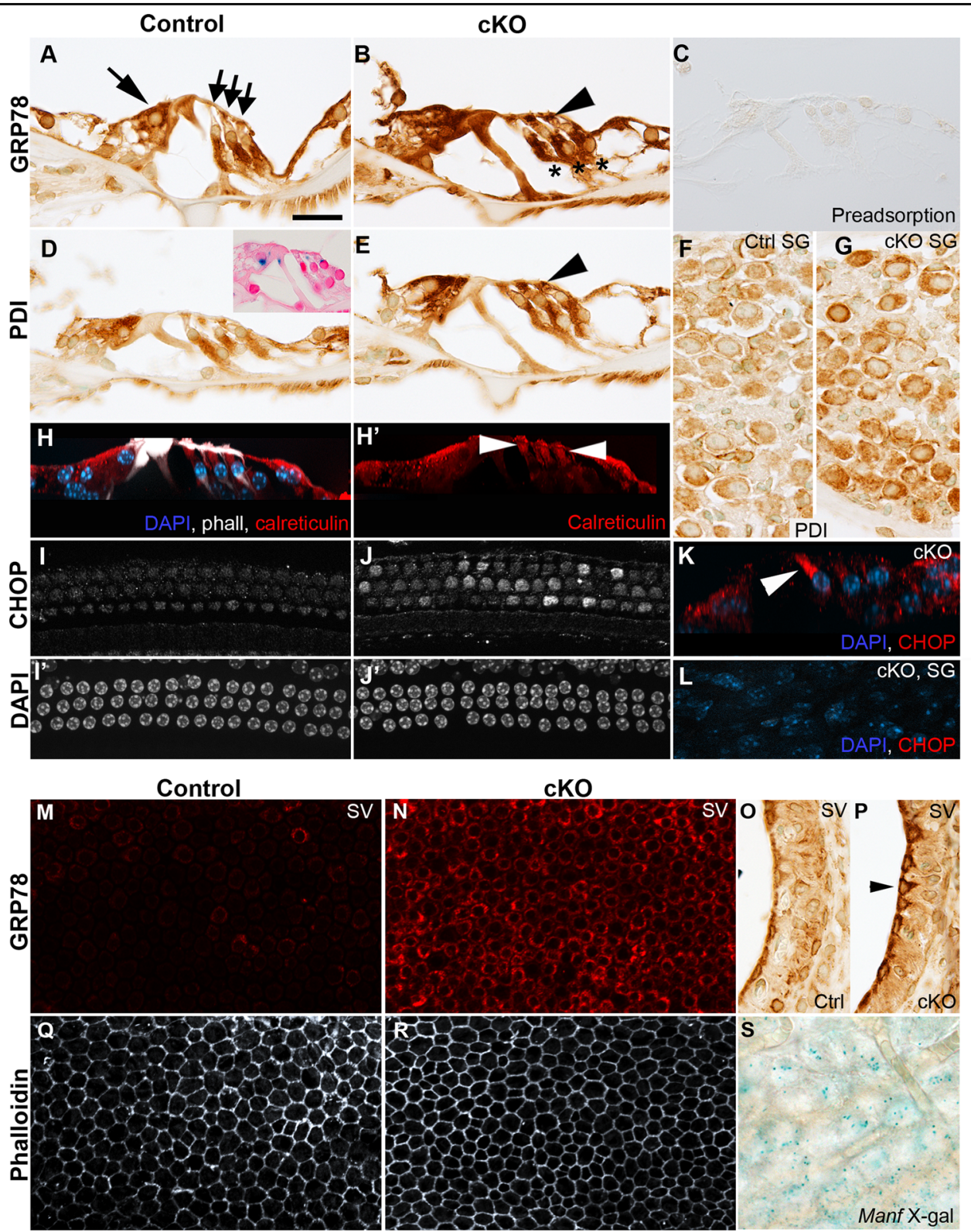

Fig. 4 Manf inactivation triggers ER stress and UPR activation in the cochlea. Cochleas from 8-week-old Manfloxflox; Pax2-Cre mice and control littermates were analyzed. Images were taken from the middle $\mathbf{a}-\mathbf{I}$ or basal part of the cochlea $\mathbf{m}-\mathbf{s}$. $\mathbf{a}, \mathbf{b}$ Transverse paraffin sections show ubiquitous GRP78 expression in the organ of Corti of control mice and GRP78 upregulation in CKOs. In OHCs, GRP78 is upregulated in the area below the cuticular plate (arrowhead). Large arrow marks IHC and small arrows OHCs. Deiters' cells beneath OHCs are marked with stars. c GRP78 antibody was validated by preadsorption assay (see the section "Materials and methods"). $\mathbf{d}$, e PDI shows also ubiquitous expression in controls and upregulation in CKOs. In OHCs, PDI upregulation has similar spatial characteristics (arrowhead) as GRP78. Inset in $\mathbf{d}$ is a reminder of Manf expression, shown by X-gal staining (see also Fig. 1). $\mathbf{f}, \mathbf{g}$ Also SG neurons of CKO cochleas show PDI upregulation, revealed in paraffin sections. $\mathbf{h}, \mathbf{h}^{\prime}$ The control whole mount is immunolabeled for calreticulin (red) and the maximum intensity projection is viewed in a transverse plane. Calreticulin expression pattern in OHCs (arrowheads) is comparable to GRP78 and PDI. Phalloidin (white) labels hair cell apices and pillar cells. DAPI labels nuclei (blue). $\mathbf{i}, \mathbf{i}^{\prime}, \mathbf{j}, \mathbf{j}^{\prime}$ Whole mount specimens show CHOP upregulation in OHCS of CKO cochleas. DAPI marks nuclei. $\mathbf{k}$ In the transverse section of a maximum intensity projection, CHOP expression (red) is seen in the first-row OHC. I Whole mount specimen from a cKO mouse shows absence of CHOP staining in SG neurons. DAPI marks nuclei (blue). $\mathbf{m}, \mathbf{n}$ Stria vascularis whole mounts reveal GRP78 upregulation in marginal cells in cKO mice. o, $\mathbf{p}$ This upregulation in marginal cells (arrowhead) is also shown in paraffin sections. $\mathbf{q}, \mathbf{r}$ Phalloidin labeling shows a regular hexagonal pattern of marginal cell boundaries, without signs of abrogated survival of these cells in CKO mice. $\mathbf{s}$ X-gal staining in a stria vascularis whole mount, viewed at the level of marginal cells, shows Manf expression (see also Fig. 1). Abbreviations: CKO conditional knock out; ctrl control; IHC inner hair cell; OHCs outer hair cells; ER endoplasmic reticulum; UPR unfolded protein response; SG spiral ganglion; GRP78 glucose-regulated protein 78; PDI protein disulfide isomerase; CHOP C/EBP homologous protein; SV stria vascularis. Scale bar (in a): a-e, h-j', I $20 \mu \mathrm{m} ; \mathbf{f}, \mathbf{g} 25 \mu \mathrm{m} ; \mathbf{k} 15 \mu \mathrm{m} ; \mathbf{m}, \mathbf{n}, \mathbf{q}, \mathbf{r} 37 \mu \mathrm{m} ; \mathbf{0}, \mathbf{p} 6 \mu \mathrm{m} ; \mathbf{s} 12 \mu \mathrm{m}$. 
resulting in phagocytosis of the apoptotic cell fragments by neighboring supporting cells. These results indicate that $\mathrm{OHC}$ death is driven from the "reactive" neck region. Interestingly, IHCs do not show this kind of subcellular ER and mitochondria aggregation. Rather, these organelles are broadly distributed in the IHC cytoplasm ${ }^{30}$. This was also revealed in our MANF and GRP78 immunostainings. These differences might contribute to the differential vulnerability of IHCs and OHCs. IHCs are known to be more death-resistant than OHCs.

Not all OHCs were lost in Manf-inactivated cochleas; it was the high-frequency OHCs in the basal part of the cochlea that were the most sensitive. It is known that they possess differences in calcium-handling capacity compared to the low-frequency $\mathrm{OHCs}$ in the upper part of the cochlea $^{29}$. In cells in general, ER machinery is a major regulator of intracellular calcium homeostasis, and ER stress is associated with elevated cytoplasmic calcium levels ${ }^{10}$. The high-frequency $\mathrm{OHCs}$ are most vulnerable to environmental stressors, such as noise and ototoxic drugs ${ }^{29}$. Thereby, there might be differences in the ER stress induction and in the UPR pathway function that dictate the calcium-dependent differential vulnerability of the highfrequency versus low-frequency $\mathrm{OHCs}$.

The stria vascularis is responsible for the endolymph ion homeostasis that it is critical for OHC survival ${ }^{31}$. MANF was weakly expressed in the strial marginal cells, and GRP78 was upregulated in these cells of Manf-inactivated cochleas. However, the survival of marginal cells was unaffected. Although our results point to cell-intrinsic regulation of $\mathrm{OHC}$ death in Manf-inactivated cochleas, we cannot exclude the possibility that ER stress impairs the ion secretory function of marginal cells, possibly then potentiating the vulnerability of ER-stressed OHCs.

In the Manf $\mathrm{KO}$ mouse line under the hybrid background $(\mathrm{CD}-1 \times \mathrm{CBA} / \mathrm{Ca})$, $\mathrm{KO}$ individuals formed two non-overlapping groups when $\mathrm{OHC}$ loss and $\mathrm{ABR}$ thresholds were analyzed. These auditory parameters were unaffected in one group, while they were strongly affected in the other group. The hybrid background appears to underlie these differences. Our results suggest that Manf inactivation alone does not trigger $\mathrm{OHC}$ loss. Rather, Manf deficiency becomes detrimental when accompanied by gene mutations that predispose to hearing loss. Several mouse strains show early-onset hearing loss, starting in the high-frequency region and gradually extending to the low-frequency region of the cochlea. In the BL6 mice, mutations in the cadherin 23 (Cdh23) gene, coding for a stereociliary tip-link protein, are known to be a major cause of age-related $\mathrm{OHC}$ loss and hearing $\operatorname{loss}^{32}$. When a Cdh23 mutation was induced in the zebrafish hair cells, it triggered ER stress and resulted in hair cell death ${ }^{33}$. A later study with the mouse cochlea supported these results by showing that $C d h 23$ mutation triggers ER stress-mediated death of $\mathrm{OHCs}^{34}$. In our Manf cKO mouse line under the BL6 background, all mutants displayed prominent $\mathrm{OHC}$ loss and hearing loss at ages prior to the onset of age-related hearing loss that characterizes this strain. This is consistent with the idea that the $C d h 23$ mutation predisposes to Manf inactivation-induced hearing loss, mechanistically by potentiating ER stress. Similar to the BL6 mice, the CD-1 mouse strain shows early-onset hearing loss, yet the underlying gene mutations are uncharacterized. In contrast, the $\mathrm{CBA} / \mathrm{Ca}$ strain does not suffer from age-related hearing loss. Therefore, in the Manf KO line under the CD-1 x CBA/Ca hybrid background, the $\mathrm{KO}$ individuals with the altered auditory parameters seem to possess the CD-1 genetic background, which intensifies ER dyshomeostasis in OHCs. This seems not to be the case with the unaffected KOs under the CBA/Ca background.

Of the NTF family members, MANF (this study) and the neurotrophins neurotrophin-3 and brain-derived neurotrophic factor promote the survival of cochlear cells, yet the site of action and mechanism of action are different. Neurotrophins are secreted from hair cells and they critically support the survival of developing SG neurons, by binding to neuronal plasma membrane receptors ${ }^{35}$. MANF is also expressed in hair cells, but it does not have a major role on SG neuron survival, not at least during the time frame used in our study. Also, the afferent innervation to OHCs appeared normal in Manf-inactivated mice, suggesting that the primary defect is at the OHC level. Furthermore, we did not find any obvious role for MANF during morphogenesis of the inner ear sensory structures. We found that MANF regulates OHC survival after the onset of hearing function, by promoting ER homeostasis in a cell-intrinsic fashion. There is evidence that MANF is secreted from cells and that it acts non-cell-intrinsically via still unidentified plasma membrane receptors ${ }^{7,13,14}$. We did not find direct evidence for this role in the cochlea. However, a putative secreted role of MANF might be linked with synaptopathy that we observed in Manf-inactivated cochleas or with the supporting cells that might regulate $\mathrm{OHC}$ survival by their MANF secretion.

Our results have translational implications. Zebrafish studies have suggested that ER stress is a proximal cause of the death of hair cells carrying gene mutations linked to the Usher syndrome, such as the Cdh23 mutation ${ }^{33}$. Usher syndrome is a leading hereditary deafness syndrome in humans ${ }^{36}$. Furthermore, ER stress and UPR activation have been suggested to mediate noise-induced hearing loss in mice ${ }^{20}$. Similarly as proposed in Parkinson's disease and diabetes ${ }^{37}$, MANF therapy might antagonize ER stress and thereby it could reduce or delay OHC death triggered by stressors that target the ER machinery. 


\section{Acknowledgements}

This work was funded by Genelec Inc., Instrumentarium Science Foundation, Finnish Association of Otorhinolaryngology and Head and Neck Surgery and Magnus Ehrnrooth Foundation (U.P.), Jenni and Antti Wihuri foundation (A.H.), Jane and Aatos Erkko Foundation (M.S.), the Academy of Finland (Grant no. 310891, M.S.; no. 117044, M.S. M.L.), and NIA R01 AG060501 (B.F.). We acknowledge Jukka Ylikoski for discussions and comments, Sanna Sihvo for excellent technical assistance, and NIH KOMP for the MANF-targeted ESC clone used to generate the Manf KO mice. MANF antibody was a kind gift from Icosagen Inc.

\section{Author details}

'Molecular and Integrative Biosciences Research Programme, University of Helsinki, 00014 Helsinki, Finland. Institute of Biotechnology, HILIFE Unit, University of Helsinki, 00014 Helsinki, Finland. ${ }^{3}$ Department of Biology, CLAS, University of lowa, lowa City, IA 52242-1324, USA

\section{Conflict of interest}

M.S. and M.L. are inventors in the MANF-related US patent (9592270B2, Neurotrophic factor MANF and uses thereof) that is owned by Herantis Pharma Plc. M.S. is a shareholder in this company.

\section{Publisher's note}

Springer Nature remains neutral with regard to jurisdictional claims in published maps and institutional affiliations.

Supplementary Information accompanies this paper at (https://doi.org/ 10.1038/s41419-020-2286-6).

\section{Received: 13 November 2019 Revised: 16 January 2020 Accepted: 17} January 2020

Published online: 06 February 2020

\section{References}

1. Petrova, P. et al. MANF: a new mesencephalic, astrocyte-derived neurotrophic factor with selectivity for dopaminergic neurons. J. Mol. Neurosci. 20, 173-188 (2003).

2. Voutilainen, M. H. et al. Mesencephalic astrocyte-derived neurotrophic factor is neurorestorative in rat model of Parkinson's disease. J. Neurosci. 29, 9651-9659 (2009).

3. Airavaara, M. et al. Widespread cortical expression of MANF by AAV serotype 7: localization and protection against ischemic brain injury. Exp. Neurol. 225, 104-113 (2010).

4. Lindahl, M. et al. MANF is indispensable for the proliferation and survival of pancreatic $\beta$ cells. Cell Rep. 7, 366-375 (2014).

5. Bell, P. A. et al. Mesencephalic astrocyte-derived neurotropic factor is an important factor in chondrocyte ER homeostasis. Cell Stress Chaperone 24, 159-173 (2019).

6. Mizobuchi, N. et al. ARMET is a soluble ER protein induced by the unfolded protein response via ERSE-II element. Cell Struct. Funct. 32, 41-50 (2007).

7. Glembotski, C. C. et al. Mesencephalic astrocyte-derived neurotrophic factor protects the heart from ischemic damage and is selectively secreted upon sarco/endoplasmic reticulum calcium depletion. J. Biol. Chem. 287, 25893-25904 (2012).

8. Yan, Y., Rato, C., Rohland, L., Preissler, S. \& Ron, D. MANF antagonizes nucleotide exchange by the endoplasmic reticulum chaperone BiP. Nat. Commun. 10, 541 (2019)

9. Walter, P. \& Ron, D. The unfolded protein response: from stress pathway to homeostatic regulation. Science 334, 1081-6 (2011).

10. Carreras-Sureda, A., Pihán, P. \& Hetz, C. Calcium signaling at the endoplasmic reticulum: fine-tuning stress responses. Cell Calcium 70, 24-31 (2018).

11. Hellman, M. et al. Mesencephalic astrocyte-derived neurotrophic factor (MANF) has a unique mechanism to rescue apoptotic neurons. J. Biol. Chem. 286, 2675-2680 (2011).

12. Mätlik, K. et al. Role of two sequence motifs of mesencephalic astrocytederived neurotrophic factor in its survival-promoting activity. Cell Death Dis. $\mathbf{6}$, e2032 (2015).
13. Henderson, M. J., Richie, C. T., Airavaara, M., Wang, Y. \& Harvey, B. K. Mesencephalic astrocyte-derived neurotrophic factor (MANF) secretion and cell surface binding are modulated by KDEL receptors. J. Biol. Chem. 288, 4209-4225 (2013).

14. Trychta, K. A., Bäck, S., Henderson, M. J. \& Harvey, B. K. KDEL receptors are differentially regulated to maintain the ER proteome under calcium deficiency. Cell Rep. 25, 1829-1840.e6 (2018).

15. Shone, G., Raphael, Y. \& Miller, J. M. Hereditary deafness occurring in CD/1 mice. Hear. Res. 57, 153-156 (1991).

16. Le Calvez, S., Avan, P., Gilain, L. \& Romand, R. CD1 hearing-impaired mice. I: Distortion product otoacoustic emission levels, cochlear function and morphology. Hear. Res. 120, 37-50 (1998).

17. Hequembourg, S. \& Liberman, M. C. Spiral ligament pathology: a major aspect of age-related cochlear degeneration in C57BL/6 mice. J. Assoc. Res. Otolaryngol. 2, 118-129 (2001).

18. Ohlemiller, K. K., Jones, S. M. \& Johnson, K. R. Application of mouse models to research in hearing and balance. J. Assoc. Res. Otolaryngol. 17, 493-523 (2016).

19. Fujinami, Y., Mutai, H., Mizutari, K, Nakagawa, S. \& Matsunaga, T. A novel animal model of hearing loss caused by acute endoplasmic reticulum stress in the cochlea. J. Pharm. Sci. 118, 363-372 (2012).

20. $L i$, J. et al. Deletion of Tmtc 4 activates the unfolded protein response and causes postnatal hearing loss. J. Clin. Invest. 128, 5150-5162 (2018).

21. Ohyama, T. \& Groves, A. K. Generation of Pax2-Cre mice by modification of a Pax2 bacterial artificial chromosome. Genesis 38, 195-199 (2004).

22. Anttonen, T. et al. How to bury the dead: elimination of apoptotic hair cells from the hearing organ of the mouse. J. Assoc. Res. Otolaryngol. 15, 975-992 (2014).

23. Herranen, A., Ikäheimo, K., Virkkala, J. \& Pirvola, U. The stress response in the non-sensory cells of the cochlea under pathological conditions-possible role in mediating noise vulnerability. J. Assoc. Res. Otolaryngol. 19, 637-652 (2018).

24. Müller, M., von Hünerbein, K., Hoidis, S. \& Smolders, J. W. Physiological placefrequency map of the cochlea in the CBA/J mouse. Hear. Res. 202, 63-73 (2005).

25. Mammano, F. et al. ATP-Induced $\mathrm{Ca}(2+)$ release in cochlear outer hair cells: localization of an inositol triphosphate-gated $\mathrm{Ca}(2+)$ store to the base of the sensory hair bundle. J. Neurosci. 19, 6918-6929 (1999).

26. Castellano-Muñoz, M. \& Ricci, A. J. Role of intracellular calcium stores in hair-cell ribbon synapse. Front. Cell. Neurosci. 8, 162 (2014).

27. Kersigo, J. \& Fritzsch, B. Inner ear hair cells deteriorate in mice engineered to have no or diminished innervation. Front. Aging Neurosci. 7, 33 (2015).

28. Maison, S., Liberman, L. D. \& Liberman, M. C. Type II cochlear ganglion neurons do not drive the olivocochlear reflex: re-examination of the cochlear phenotype in peripherin knock-out mice. eNeuro 3, https:/doi.org/10.1523/ ENEURO.0207-16.2016 (2016).

29. Fettiplace, R. \& Nam, J. H. Tonotopy in calcium homeostasis and vulnerability of cochlear hair cells. Hear. Res. 376, 11-21 (2019).

30. Bullen, A. et al. Association of intracellular and synaptic organization in cochlear inner hair cells revealed by 3D electron microscopy. J. Cell Sci. 128 2529-2540 (2015)

31. Liu, H. et al. Organ of Corti and stria vascularis: is there an interdependence for sunvival? PLOS ONE 11, e0168953 (2016)

32. Johnson, K. R., Zheng, Q. Y. \& Noben-Trauth, K. Strain background effects and genetic modifiers of hearing in mice. Brain Res. 1091, 79-88 (2006).

33. Blanco-Sánchez, B., Clément, A., Fierro, J. Jr., Washbourne, P. \& Westerfield, M. Complexes of usher proteins preassemble at the endoplasmic reticulum and are required for trafficking and ER homeostasis. Dis. Models Mech. 7, 547-759 (2014).

34. $\mathrm{Hu}$, J. et al. ER stress inhibitor attenuates hearing loss and hair cell death in Cdh23erl/erl mutant mice. Cell Death Dis. 7, e2485 (2016).

35. Fritzsch, B., Pirvola, U. \& Ylikoski, J. Making and breaking the innervation of the ear: neurotrophic support during ear development and its clinical implications. Cell Tissue Res. 295, 369-382 (1999).

36. Bonnet, C. \& El-Amraoui, A. Usher syndrome (sensorineural deafness and retinitis pigmentosa): pathogenesis, molecular diagnosis and therapeutic approaches. Curr. Opin. Neurol. 25, 42-49 (2012).

37. Lindahl, M., Saarma, M. \& Lindholm, P. Unconventional neurotrophic factors CDNF and MANF: structure, physiological functions and therapeutic potential. Neurobiol. Dis. 97, 90-102 (2017). 